\title{
Location of art gallery in urban areas: A GIS based analysis in Shinagawa ward, Tokyo
}

\author{
Yuta TSUNEIZUMI ${ }^{\text {a, *, Kaori ITO }}{ }^{\text {a }}$, Seiya TAKAYANAGI ${ }^{\text {a }}$ \\ a Tokyo University of Science, Japan; tsuney.762@gmail.com,kaori@rs.tus.ac.jp,seiya@rs.tus.ac.jp
}

Keywords: art gallery, spatial analysis, Ripley’s K function, GIS

\begin{abstract}
:
In recent years there has been increasing support for creative industry in various countries. Especially, art and artist play an important role in the creative city. For example, in New York's SoHo (South of Houston) neighborhood, artists created new methods of urban development in the 1960s by using their labor and design expertise to invent a new form of housing, the loft apartment, in former industrial space (Shkuda, 2015). In such cases, the artist transforms a facility such as a warehouse, into a gallery or atelier. After that, we can experience art activities or purchase art works in galleries in the city. How easily citizen can access art and culture is much important when we think the creative city and make new urban policies for artists and citizen. However, many of research about creative industries aren't focuses on art environment. In order to clarify the environment where artists gather, it is necessary to investigate where the art gallery is more likely to be located than other facilities. To think about these problems, this research aims to clarify the characteristic of the location of art gallery by comparing other type facilities like warehousing business.
\end{abstract}

The study was conducted urban area of Japan (Tokyo 23 Wards and Osaka 24 Wards). To conduct our research, we used "The fine arts \& contemporary arts data book 2021" (Seikatsu no Tomo-sha, 2021), \& Digital yellow pages (telepoint data) provided NTT. And we collected two category data, "private art gallery" and "warehousing business office". After the plotting data on QGIS, we analyzed spatial distribution pattern of art gallery with point pattern analysis considering spatial dependency.

The analysis of spatial distribution was performed using Ripley's K function. Ripley's K function is a density probability function that permits a second-order analysis. It is based on the variance of all point-to-point distances. The procedure comprises delimitating a circle of radius $h$ centered on each point and counting the number of neighbors within this circle. By varying the radius $h$, it is possible to determine the pattern at different distance scales. To facilitate the visualization of the results, the $\mathrm{K}(\mathrm{h})$ function was transformed into an $\mathrm{L}(\mathrm{h})$ function. We used $\mathrm{R}$ software and mainly used the package Splancs. Simulation envelopes were calculated using 99 Monte Carlo simulations. If the spatial pattern is completely random, the L(h) function will appear as a line within confidence intervals. The relationship of dependence or independence between art gallery and warehousing business base was analyzed using the bivariate $\mathrm{K}$ function. In the bivariate analysis, by comparing observed difference of $\mathrm{K}$ values, it can be clarified whether one is more concentrated than the other.

In this paper, we present the analysis of Shinagawa Ward, Tokyo as a case study (Figure 1). In Shinagawa Ward, some art facilities converted from warehouse to art gallery are located.

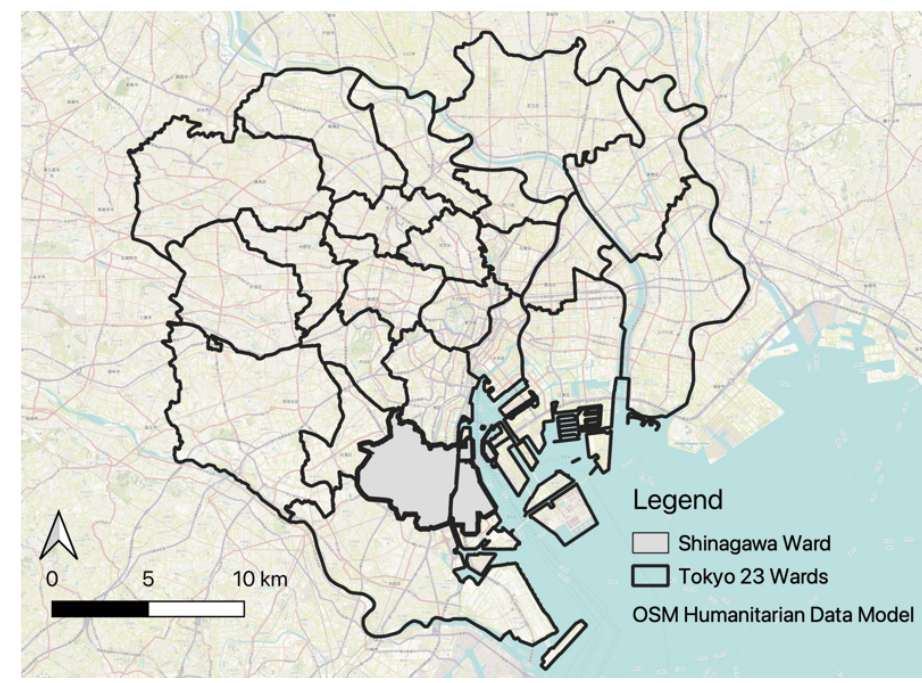

Figure 1. Location of Shinagawa Ward 
As a result, in Shinagawa Ward, we found 20 art gallery and 44 warehousing business office (figure 2). According to the bivariate $\mathrm{K}$ function plots, the distribution of galleries were more clustered lower than $2.0 \mathrm{~km}$ (figure 3 ). On the other hand, $\mathrm{K}$ function analyses also indicated that art gallery and warehousing business base were independently spatial distributed at distances greater than $2.0 \mathrm{~km}$.

Since some art facilities converted from warehouse to art galleries are located in waterfront area, it is possible that the result is that the art galleries are highly concentrated within the range of $2.0 \mathrm{~km}$. However, the results of this analysis may include the impact of characteristic urban development. In the future, we need to conduct surveys in wider areas.

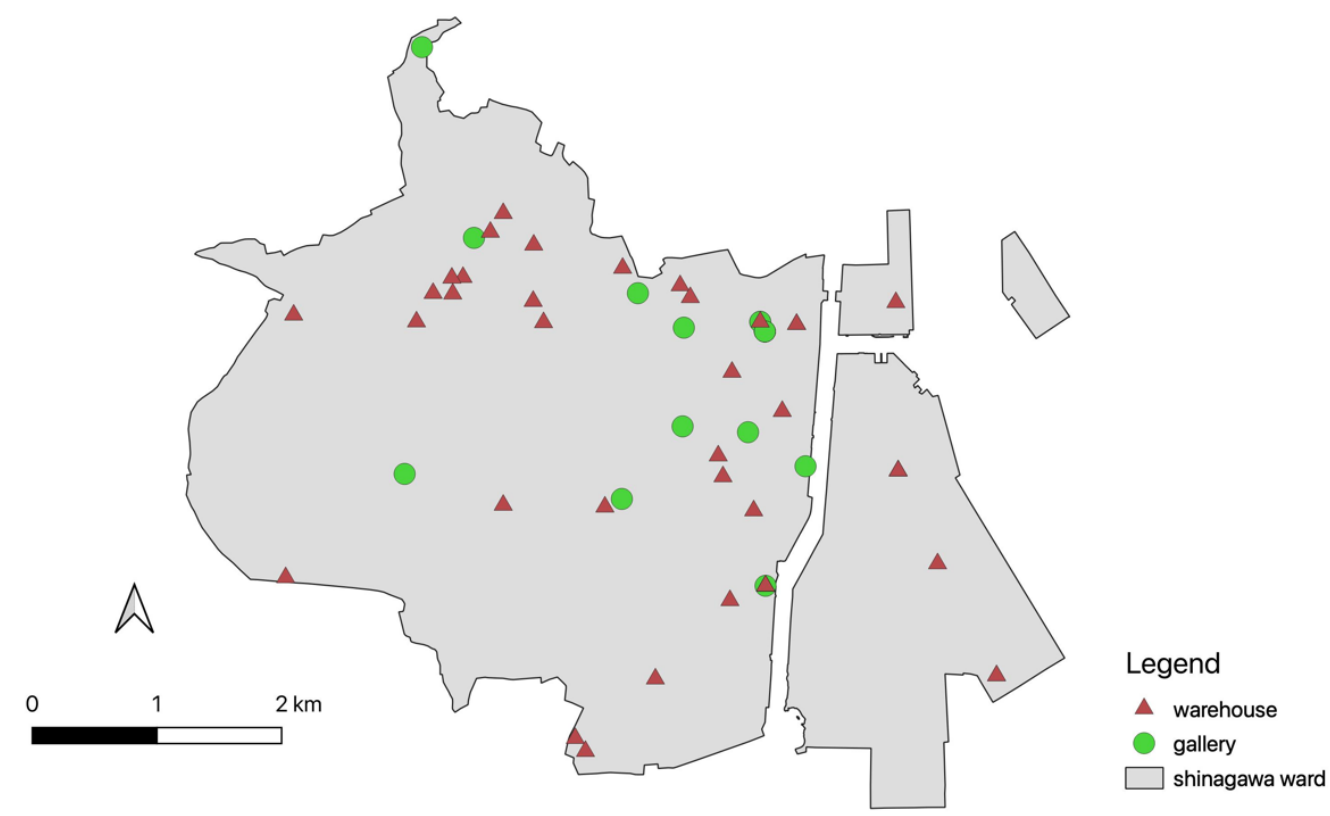

Figure 2. Distribution map of the art gallery and the warehousing business office

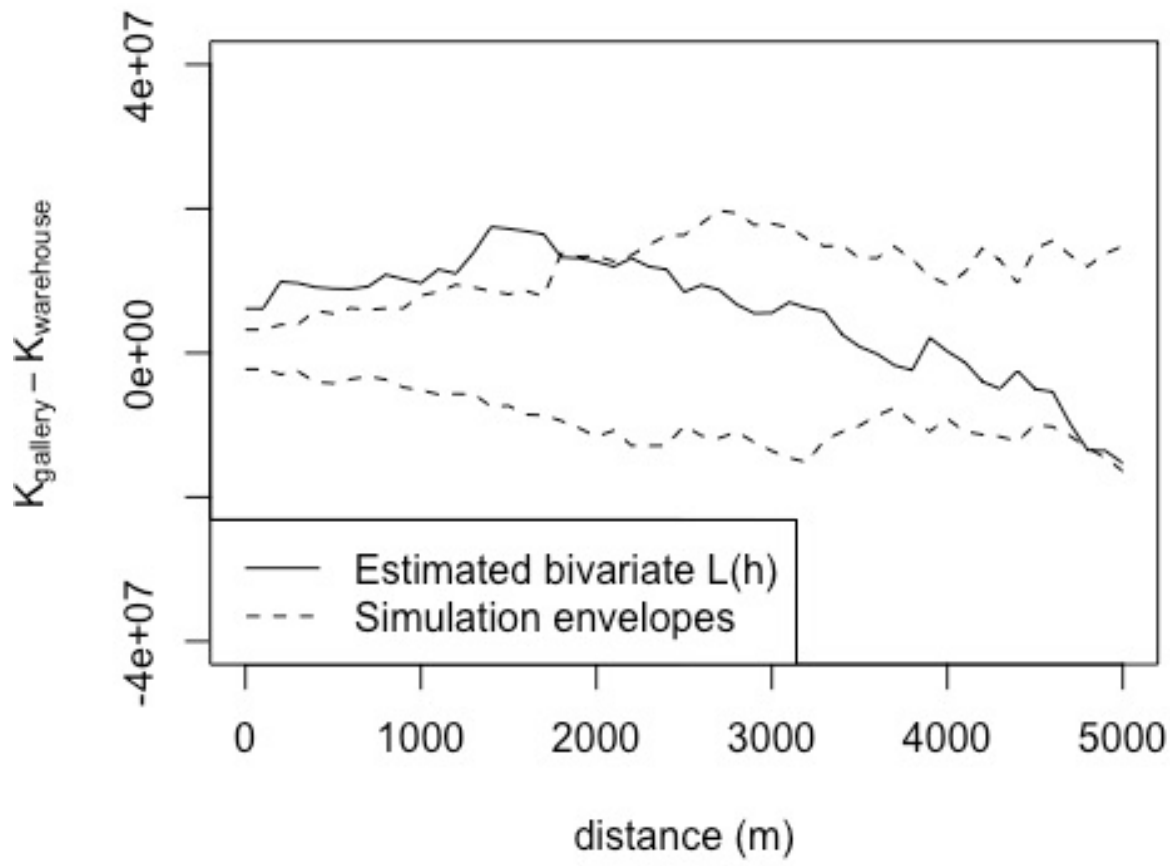

Figure 3. Bivariate Ripley’s K function analysis comparing the spatial distribution 